(C) 2015 IEEE. Personal use of this material is permitted. Permission from IEEE must be obtained for all other uses, in any current or future media, including reprinting/republishing this material for advertising or promotional purposes, creating new collective works, for resale or redistribution to servers or lists, or reuse of any copyrighted component of this work in other works. 


\title{
Predictive Control Algorithm to Achieve Power Balance of Cascaded H-Bridge Converters
}

\author{
Ricardo P. Aguilera*, Yifan Yu*, Pablo Acuna*, Georgios Konstantinou*, Christopher D. Townsend*, \\ Bin $\mathrm{Wu}^{\dagger}$, Vassilios G. Agelidis* \\ *Australian Energy Research Institute, University of New South Wales, Australia, \\ ${ }^{\dagger}$ Department of Electrical and Computer Engineering, Ryerson University, Canada. \\ Email: raguilera@ieee.org
}

\begin{abstract}
This work proposes a Model Predictive Control (MPC) strategy for Cascaded H-Bridge (CHB) converters under unbalanced power generation among each converter phase. Therefore, the control target is to extract unbalanced power from the dc-sources while providing balanced power to the grid. The key novelty of this proposal lies in the way the unbalanced power generation issue is explicitly considered into the optimal control problem. The power balance is achieved by enforcing the CHB to work with a suitable zero voltage components. Thus, to account for the common-mode voltage, the proposed MPC is directly formulated in the original $a b c$-framework . To verify the effectiveness of this proposal, simulation results of the proposed MPC governing a five-level CHB converter are provided.
\end{abstract}

Index Terms-Multilevel converters, cascaded H-bridge converters, DC-AC power converters, control design, predictive control, finite control set, power distribution.

\section{INTRODUCTION}

Multilevel converters (MCs) are an interesting technology for medium/high-voltage and high power applications [1]. Several MCs have been proposed in the literature, which offer different features. Despite the difference in their topologies, MC have a common feature: they are able to produce high quality voltage and current waveforms at medium/high-voltage range by using power switches rated at lower voltage values. To do this, special modulation and/or control techniques are used to distribute de voltage (and, hence, power) among the internal power switches. This has allowed power converters to work in high power applications, such as high-power drives [2], active filters [3], renewable energy grid integration [4], etc. Among the different MCs one can find in the literature, the most popular and widely used are: the Neutral Point Clamped (NPC) [3], Flying Capacitor (FC) [5], Cascaded HBridge (CHB) [6], and Modular Multilevel Converter (MMC) [7]. This work is particularly focused on the control of a CHB converter. This topology is comprised of a basic unit called cell, which is a simple three-level HB converter. Thus, to extend the voltage levels of the whole converter, several cells are interconnected in series. To obtain the total output voltage, each cell is electrically fed with isolated dc voltage sources. This makes this topology particularly suitable for the integration of solar PV plants to the electricity grid [4]. Due to the large extension of these plants, they are likely to be affected by partial shading. Therefore, PV strings may deliver different amount of maximum power.
To address this issue, several control strategies have been proposed to govern $\mathrm{CHB}$ converters under unequal power generation among phases [8]-[10]. In this case, the control goal is to achieve an inter-phase power balance in the gridside under a certain range of power imbalance in the dc-power source. Existing approaches are aimed to obtain symmetric sinusoidal currents by using standard PI controllers in a $d q$ framework. Thus, the $d q$-voltage provided by the controller is transformed into the $a b c$-framework obtaining a symmetrical converter voltage reference, $v_{a b c}^{+}$. To achieve the inter-phase power balance in the grid side, it is possible to add a suitable zero-sequence component, $v^{0}$, to the symmetrical component reference, i.e., $v_{a b c}=v_{a b c}^{+}+v^{0}$. Then, a PWM stage is used to synthesize this references.

Regarding modern control strategies for power converters, Model Predictive Control (MPC) has emerged as an interesting alternative to govern power converters [11], [12]. The main advantage of MPC comes from the fact that system constraints (e.g., current and voltage admissible levels, and switch states) and nonlinearities can be explicitly considered in the optimization [13]. Different predictive control formulations have been proposed to govern power converters, showing that these methods, in general, may outperform standard PWMbased controllers. Due to its flexibility and potentiality, Finite Control Set MPC (FCS-MPC) is one of the most popular predictive controller for power converters [11], [14]. FCS-MPC directly considers the power switches (or voltage levels) in the optimization as input constraints [13]. Thus, no modulation stages are needed. To obtain the optimal solution, one can evaluate all the possible switching combinations in the cost function and then apply the one which minimizes it. Some examples of recent predictive control formulations in power electronics can be found in [11]-[20].

Motivated by the above, an FCS-MPC for CHB converters under unbalanced power generation is proposed in this work. This is designed to extract unbalanced power from the dcsources in a CHB converter while providing balance power to the grid. To account for the common-mode voltage, the proposed predictive controller is directly formulated in the original $a b c$-framework. This allows the controller to track balanced current with an asymmetric inverter voltage, which ensures an inter-phase power balance. To verify the effectiveness and performance of this proposal, simulation results of a 
three-phase two-cell CHB converter governed by the proposed predictive control strategy are provided.

\section{CHB CONVERTER MOdEL}

The CHB topology is comprised of a basic unit called cell, which is a simple three-level HB converter. Thus, to extend the voltage levels of the whole converter, several cells are interconnected in series. To obtain the total output voltage, each cell is electrically fed with isolated dc voltage sources as shown in Fig. 1.

Considering a grid-connected $\mathrm{CHB}$ converter, by using simple circuit analysis, the following continuous-time dynamic equation for each phase current can be obtained:

$$
\frac{d i_{y}(t)}{d t}=-\frac{r}{L} i_{y}(t)+\frac{1}{L}\left(v_{y}(t)-v_{g y}(t)-v_{N o}(t)\right)
$$

for all $y \in\{a, b, c\}$. Parameters $r$ and $L$ stand for the resistance and inductance of the output filter. Moreover, $v_{g y}(t)$ represents the grid voltage per phase, while

$$
v_{y}(t)=\sum_{j=1}^{n} v_{y j}(t)
$$

stands for the total converter output voltage per phase, in which $v_{y j}(t)$ is the individual output cell voltage, and $n$ is the number of cells per phase. Additionally, $v_{N o}$ is the, socalled, common-mode voltage, which is given by

$$
v_{N o}=\frac{1}{3}\left(v_{a}(t)+v_{b}(t)+v_{c}(t)\right) .
$$

\section{A. Control Input}

In general, when using FCS-MPC for governing power converters, it is common to consider the converter power switches as control input [13]. When doing this, the output voltage of each $\mathrm{H}$-Bridge cell can be defined as

$$
v_{y j}=V_{d c}\left(S_{y j 1}-S_{y j 2}\right) .
$$

where $y \in\{a, b, c\}$ and $j \in\{1, \ldots, n\}$. Since each power switch can adopt only two states, i.e., $S_{y j 1} \in\{0,1\}$, this results in $2^{2}$ switching combination per cell. Therefore, taking into account (2), for a three-phase $n$-cell CHB converter, the total number of switch combinations is given by

$$
N_{S C}=2^{6 n} \text {. }
$$

Thus, for the particular case of a two-cell CHB converter, a total of 4096 input (power switch) combinations is obtained. This represents a practical implementation problem of the predictive controller, since it requires to evaluate all these input combinations in the cost function to obtain the optimal one. To address this issue, it is possible to take advantage of the fact that some of these switch combinations generate the same output voltage per phase. Therefore, in this work, the use of the phase voltage levels as control input instead of power switches is considered. Then, the cell output voltage in (4) becomes

$$
v_{y j}=V_{d c} v_{\ell y}
$$

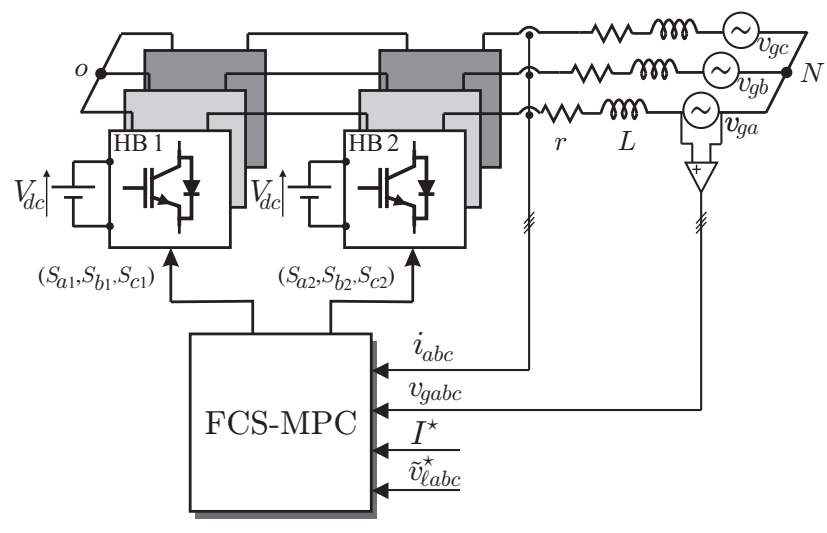

Fig. 1. CHB schematic and block diagram of the proposed FCS-MPC.

where

$$
v_{\ell y} \in \mathbb{V}=\{-n,-n+1, \ldots, 0, \ldots, n-1, n\} .
$$

With this approach, for a three-phase $n$-cell CHB converter, the number of voltage level combinations, considering (2), is given by

$$
N_{V L C}=(2 n+1)^{3},
$$

which drastically reduces the input combinations when compared to (4). For instance, for the two-cell case, we have now only 125 input (voltage level) combinations.

\section{B. Discrete-Time Model}

In this work, the implementation of an FCS-MPC for a CHB converter in the original $a b c$-framework is proposed. To achieve this, the system state is chosen as

$$
x(k)=\left[\begin{array}{l}
i_{a}(k) \\
i_{b}(k)
\end{array}\right] \in \mathbb{R}^{2},
$$

where $i_{c}(k)=-\left(i_{a}(k)+i_{b}(k)\right)$. Then, as previously mentioned, the control input is chosen as

$$
u(k)=\left[\begin{array}{l}
v_{\ell a}(k) \\
v_{\ell b}(k) \\
v_{\ell c}(k)
\end{array}\right], \in \mathbb{U},
$$

which belongs to the finite control set

$$
\mathbb{U}=\mathbb{V}^{3} .
$$

Therefore, by applying the so-called forward Euler discretization to (1) with a sampling period of $T_{s}$, the following discretetime dynamic model can be obtained:

$$
x(k+1)=A x(k)+B u(k)+E v_{g}(k)
$$

where

$$
\begin{aligned}
A & =\left[\begin{array}{ccc}
1-\frac{r T_{s}}{L} & 0 & \\
0 & 1-\frac{r T_{s}}{L}
\end{array}\right], \\
B & =\frac{V_{d c} T_{s}}{3 n L}\left[\begin{array}{ccc}
2 & -1 & -1 \\
-1 & 2 & -1
\end{array}\right], \\
E & =-\frac{T_{s}}{L} \mathrm{I}_{2 \times 2},
\end{aligned}
$$


in which

$$
v_{g}=\left[\begin{array}{c}
v_{g a}(k) \\
v_{g b}(k)
\end{array}\right]
$$

is the balanced grid voltage vector, i.e., $v_{g c}(k)=-\left(v_{g a}(k)+\right.$ $\left.v_{g b}(k)\right)$.

\section{Proposed FCS-MPC OF A CHB Converter}

In this section, the proposed FCS-MPC is formulated. Firstly, a general description of the predictive controller is given. Then, proper voltage references to achieve the power balance control target are derived.

\section{A. Basic Principle}

To implement the horizon-one FCS-MPC strategy, at each discrete instant $k$, a measurement of the system state $x(k)$ is taken and then a cost function is evaluated for each control input element in $\mathbb{U}$ as per (11). Normally, in power electronics, the cost function only penalize the current tracking error. Then, the control target is to achieve and keep a steady state reference for the output current, i.e.,

$$
x^{\star}(t)=\left[\begin{array}{c}
I^{\star} \sin (\omega t) \\
I^{\star} \sin (\omega t-2 \pi / 3)
\end{array}\right] .
$$

Thus, the standard horizon-one cost function can be expressed as (see [11]):

$$
J_{s t d}(k)=\left\|x^{\prime}(k+1)-x^{\star}(k+1)\right\|_{2}^{2},
$$

where $x^{\prime}(k+1)$ stands for the state predictions and $\left\|a-a^{\star}\right\|_{2}^{2}=$ $\left(a_{1}-a_{1}^{\star}\right)^{2}+\ldots+\left(a_{p}-a_{p}^{\star}\right)^{2}$, for a pair of vectors $a, a^{\star} \in$ $\mathbb{R}^{p}$. As shown in [21], considering only the tracking current error in the cost function leads, in general, to a high commonmode voltage since several inputs generate the same output current. To address this problem, in [21] voltage redundancies are eliminated by selecting only the voltage vectors which generate a low common-mode voltage.

Recently, in [22], an FCS-MPC design with guaranteed performance has been proposed for power converters. Here, the use of the input tracking error has been considered:

$$
J(k)=\left\|x^{\prime}(k+1)-x^{\star}(k+1)\right\|_{2}^{2}+\sigma\left\|u^{\prime}(k)-u^{\star}(k)\right\|_{2}^{2},
$$

where $u^{\prime}(k)$ is the tentative input combination that generates the prediction $x^{\prime}(k+1)$, and $u^{\star}(k)$ is the required CHB voltage to keep the current reference (14) in the steady-state. Here, $\sigma$ stands for the weighting factor that allows one to adjust a desired closed-loop performance; see [22]. Consequently, the optimal voltage level to be applied by the converter is the one that minimizes the cost function, i.e.,

$$
u^{o p}(k)=\arg \left\{\min _{u \in \mathbb{U}} J(k)\right\} .
$$

Consequently, the CHB converter, governed by (17), yields the closed-loop equation

$$
x(k+1)=A x(k)+B u^{o p}(k)+E v_{g}(k) .
$$

This procedure is repeated at each sampling instant using fresh measurements of the currents and grid voltages. In Fig. 1, a block diagram of this predictive control strategy is presented.

Notice that when the system state is near its reference, $x(k) \approx x^{\star}(k)$, the first part of the cost function is almost zero. Therefore, the second term becomes the predominant term which defines the control action. This leads to an optimal control input, $u^{o p}(k)$, that tracks the input reference, $u^{\star}(k)$, during the steady-state.

\section{B. Symmetric Voltage Reference}

Here, the aim is to obtain balance sinusoidal currents, as per (14), while maintaining a minimum possible common-mode voltage. To do so, firstly, the steady-state derivative of the current reference is obtained:

$$
\frac{d i_{y}^{\star}(t)}{d t}=\omega I^{\star} \cos \left(\omega t+\phi_{y}\right),
$$

where $\phi_{y} \in\{0,-2 \pi / 3\}$. Then, evaluating the desired steadystate condition in (1), and considering a null common-mode voltage, i.e. $v_{N o}^{\star}=0$, it follows that the required symmetrical input to keep the sinusoidal reference in (1) is given by

$v_{\ell y}^{\star}(t)=\frac{1}{V_{d c}}\left(I^{\star}\left(X_{L} \cos \left(\omega t+\phi_{y}\right)+r \sin \left(\omega t+\phi_{y}\right)\right)+v_{g y}(t)\right)$

Consequently, to achieve balanced sinusoidal currents with a reduced common-mode voltage, FCS-MPC is implemented by using the proposed cost function, $J(k)$ in (16), with $x^{\star}(k)$ as per (14) and

$$
u^{\star}(k)=\left[\begin{array}{c}
v_{\ell a}^{\star}(k) \\
v_{\ell b}^{\star}(k) \\
v_{\ell c}^{\star}(k)
\end{array}\right] .
$$

\section{Unbalanced Power Source Reference}

To highlight the benefits of the proposed cost function, $J(k)$ in (16), the control of a CHB converter with unbalanced power in the dc-source is investigated. Here, the control target is to extract different amount of power from each CHB converter phase while injecting balanced power to the grid. This case is particularly important for solar PV plants, which may be affected with partial shading, see [9]. A standard approach to achieve this control target is by enforcing a suitable zero sequence voltage in the CHB converter output [9]. Thus, the desired voltage reference can be expressed by

$$
\tilde{v}_{y}^{*}(t)=v_{y}^{+}(t)+v^{0}(t) .
$$

Then, it is assumed that the nominal power that the PV solar plant can provide, in a normal condition, is $P_{n o m}$. Then, to account for the reduction in power per phase, a power generation ratio $\lambda_{y} \in[0,1]$ is introduced, i.e., a maximum power of $\lambda_{y} P_{n o m} / 3$ can be extracted for the phase $y \in\{a, b, v\}$. Thus, the required balanced current, under a reduced power source, can be obtained as per

$$
I^{\star}=\frac{3}{2} \frac{P_{n o m}}{\hat{V}_{g}} \frac{\left(\lambda_{a}+\lambda_{b}+\lambda_{c}\right)}{3},
$$


(a)

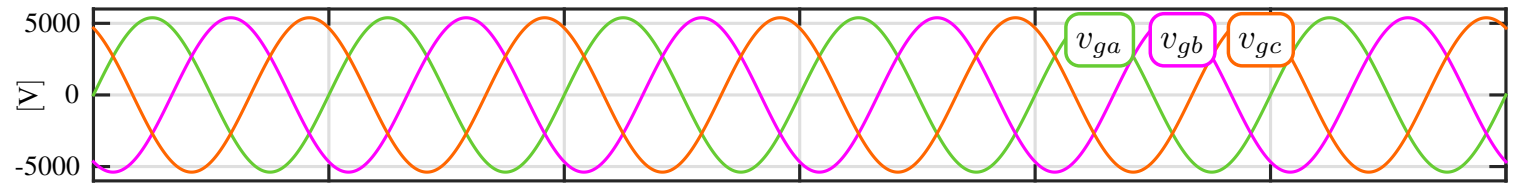

(b)

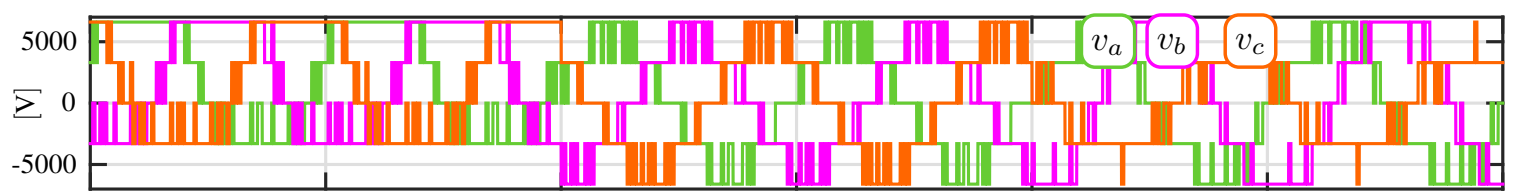

(c)

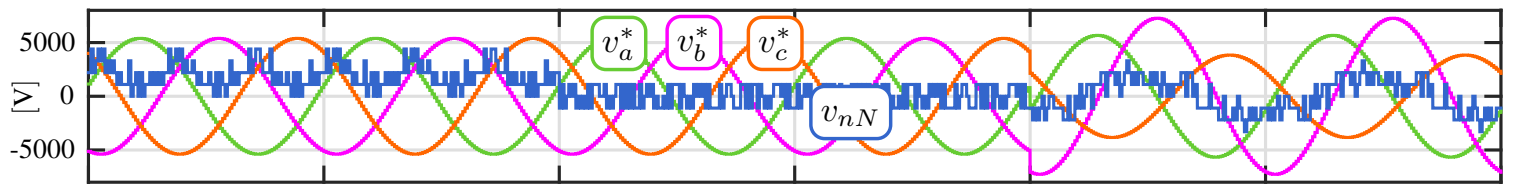

(d)

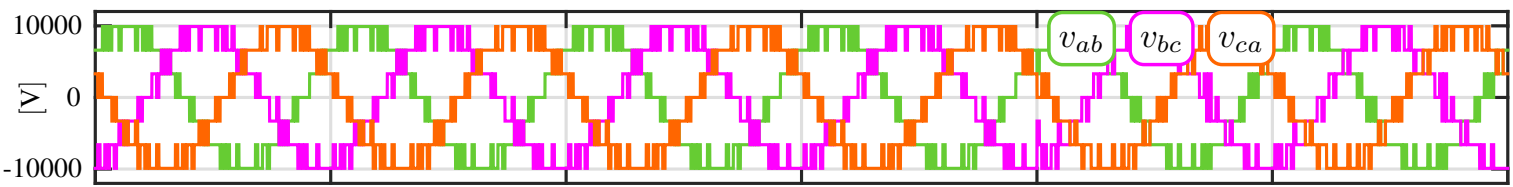

(e)

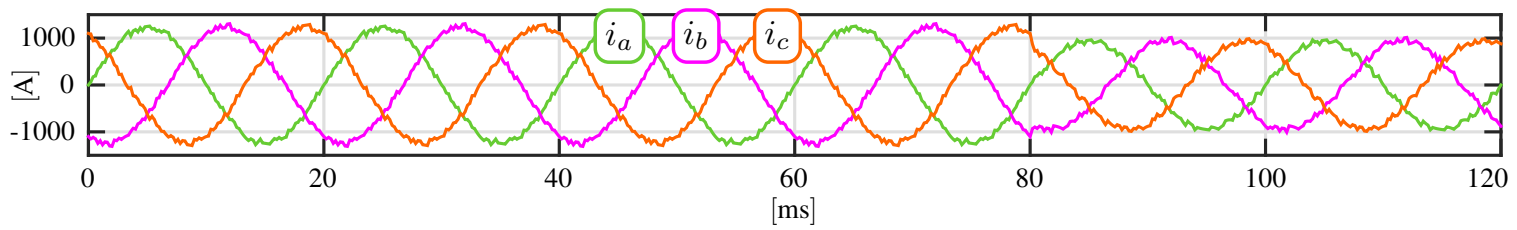

Fig. 2. Simulation results of the proposed FCS-MPC. (a) grid voltages; (b) CHB output voltages; (c) CHB voltage references; (d) CHB line-to-line voltages; (e) injected grid currents.

where $\hat{V}_{g}$ is the amplitude of the grid voltage. Therefore, the positive sequence component of the unbalanced CHB voltage reference is

$$
v^{+}(t)=V_{d c} v_{\ell y}^{\star}(t)
$$

where $v_{\ell y}^{\star}(t)$ is obtained from (20) by using (23). Finally, the required zero voltage sequence is given by

$$
v^{0}(t)=\hat{V}^{0} \sin \left(\omega t+\theta_{0}\right)
$$

where

$$
\begin{aligned}
\hat{V}^{0} & =\frac{\sqrt{6} \Delta}{3\left(\lambda_{a}+\lambda_{b}+\lambda_{c}\right)}\left(\hat{V}_{g} \sqrt{3}\right), \\
\theta_{0} & = \begin{cases}\sin ^{-1}\left(\frac{\sqrt{6}\left(\lambda_{c}-\lambda_{b}\right)}{2 \Delta}\right) & \text { Sectors (I), (VI) } \\
\frac{2 \pi}{3}+\sin ^{-1}\left(\frac{\sqrt{6}\left(\lambda_{b}-\lambda_{a}\right)}{2 \Delta}\right) & \text { Sectors (II), (III), } \\
\frac{4 \pi}{3}+\sin ^{-1}\left(\frac{\sqrt{6}\left(\lambda_{a}-\lambda_{c}\right)}{2 \Delta}\right) & \text { Sectors (IV), (V) }\end{cases}
\end{aligned}
$$

in which $\Delta=\sqrt{\left(\lambda_{a}-\lambda_{b}\right)^{2}+\left(\lambda_{b}-\lambda_{c}\right)^{2}+\left(\lambda_{a}-\lambda_{c}\right)^{2}}$. For the sake of brevity, details on how to derive the above zero sequence voltage reference are not included in this work. Further details can be found in [9].

Consequently, to extract unbalanced power from each CHB converter phase while injecting balanced power into the grid,
FCS-MPC is implemented by using the proposed cost function, $J(k)$ in (16), with $x^{\star}(k)$ as per (14) with (23), and

$$
u^{\star}(k)=\left[\begin{array}{c}
\tilde{v}_{\ell a}^{\star}(k) \\
\tilde{v}_{\ell b}^{\star}(k) \\
\tilde{v}_{\ell c}^{\star}(k)
\end{array}\right],
$$

where

$$
\tilde{v}_{\ell y}^{\star}(k)=v_{\ell y}^{\star}(k)+\frac{1}{V_{d c}} v^{0}(k) .
$$

Notice that for the balanced case when $\lambda_{a}=\lambda_{b}=\lambda_{c}$, the common-mode voltage reference becomes $v_{N o}=0$, which leads to the symmetric case presented in Section III-B, i.e., $\tilde{v}_{\ell_{y}}^{\star}=v_{\ell_{y}}^{\star}$. A block diagram of the proposed predictive control strategy is also included in Fig. 1.

It is important to emphasize that (25) is not the only viable option to obtain $v^{0}$. Any zero-sequence reference that guarantees inter-phase power balance can be applied along with the proposed predictive controller; see, e.g., [8]-[10].

\section{RESULTS}

Simulation results of a three-phase two-cell CHB converter governed by the proposed FCS-MPC are presented in this section to validate the effectiveness and performance of the proposed predictive controller. The main system parameters are presented in Table I. Main results are presented in Fig. 2.

During the first $40 \mathrm{~ms}$, the predictive controller is tuned with $\sigma=0$, which is equivalent to use the standard cost function 
Table II

Steady State Performance Results

\begin{tabular}{|c|c|c|c|c|c|c|c|c|c|}
\hline \multirow{2}{*}{$\begin{array}{l}\text { Balance case } \\
\text { Weighting factor } \\
\text { Phase }\end{array}$} & \multicolumn{3}{|c|}{$\begin{array}{c}\lambda_{a}=1, \lambda_{b}=1, \lambda_{c}=1 \\
\sigma=0\end{array}$} & \multicolumn{3}{|c|}{$\begin{array}{c}\lambda_{a}=1, \lambda_{b}=1, \lambda_{c}=1 \\
\sigma=1 e^{-6}\end{array}$} & \multicolumn{3}{|c|}{$\begin{array}{c}\lambda_{a}=0.8, \lambda_{b}=1, \lambda_{c}=0.5 \\
\sigma=1 e^{-6}\end{array}$} \\
\hline & $\mathrm{a}$ & $\mathrm{b}$ & $\mathrm{c}$ & $\mathrm{a}$ & $\mathrm{b}$ & $\mathrm{c}$ & $\mathrm{a}$ & $\mathrm{b}$ & $\mathrm{c}$ \\
\hline $\mathrm{CHB}$ phase voltage (rms) [V] & 3900.2 & 3904.2 & 3906.1 & 3920.5 & 3864.6 & 3919.5 & 4169.1 & 4993.6 & 2762.7 \\
\hline $\mathrm{THD}_{v}[\%]$ & 31.4 & 32.3 & 32.4 & 26.7 & 26.8 & 25.9 & 26.3 & 21.5 & 36.8 \\
\hline Grid current (rms) $[\mathrm{A}]$ & 875.4 & 876.1 & 874.7 & 875.2 & 875.6 & 874.2 & 666.7 & 673.8 & 671.0 \\
\hline $\mathrm{THD}_{i}[\%]$ & 3.1 & 3.2 & 3.5 & 3.1 & 3.2 & 3.5 & 4.4 & 4.9 & 5.1 \\
\hline CHB Power [MW] & 3.41 & 3.42 & 3.41 & 3.43 & 3.38 & 3.42 & 2.77 & 3.36 & 1.85 \\
\hline Total Power [MW] & & 10.24 & & & 10.23 & & & 7.98 & \\
\hline
\end{tabular}

Table I

SySTEM PARAMETERS

\begin{tabular}{ccc}
\hline \hline Variable & Description & Simulation \\
\hline$P_{n o m}$ & Three-phase nonimal power & $10 \mathrm{MW}$ \\
$v_{g}$ & Grid voltage (line-to-line rms) & $6.6 \mathrm{kV}$ \\
$f$ & Grid frequency & $50 \mathrm{~Hz}$ \\
$V_{d c}$ & dc capacitor voltage per HB & $3300 \mathrm{~V}$ \\
$L_{f}$ & Filter inductor & $3 \mathrm{mH}$ \\
$f_{s}$ & Sampling frequency & $5 \mathrm{kHz}$ \\
$f_{s w}$ & Average switching frequency per switch $272.2 \mathrm{~Hz}$ \\
$f_{v}$ & Average switching frequency of $v_{a b c}$ & $1.63 \mathrm{kHz}$ \\
\hline \hline
\end{tabular}

$J_{s t d}(k)$ in (15). From Fig. 2c, one can clearly observe that a large average value of the common-voltage is obtained. This is also reflected in the CHB phase voltages Fig. 2b. To address this issue, at the instant $t=40 \mathrm{~ms}$, the weighting factor is set to $\sigma=1 e^{-6}$. This immediately reduces the common-mode voltage to a small value centered around zero, as shown in Fig. 2b. As a result of this action, a symmetric three-phase CHB voltage is achieved, as depicted in Fig. 2b. It is important to emphasize that in both cases the same line-to-line voltage is obtained (see Fig. 2d), which produces the same output current (see Fig. 2e). This can be also observed from the data presented in Table II. In both cases the output currents present the same THD.

To verify the power balance performance of the proposed predictive controller, at the instant $t=80 \mathrm{~ms}$ a source power imbalance of $\lambda_{a}=0.8, \lambda_{a}=1$, and $\lambda_{c}=0.5$ is introduced. This yields to a step-down in the output current reference as per (23); see Fig. 2e. A zoom to the three-phase current and inverter voltage is depicted in Fig. 3. To preserve the well-known fast dynamic response of this class of predictive controller, the wighting factor is tuned with a small value; $\sigma=1 e^{-6}$ in this case. Therefore, during transients the controller has the priority of minimizing the current tracking (a)

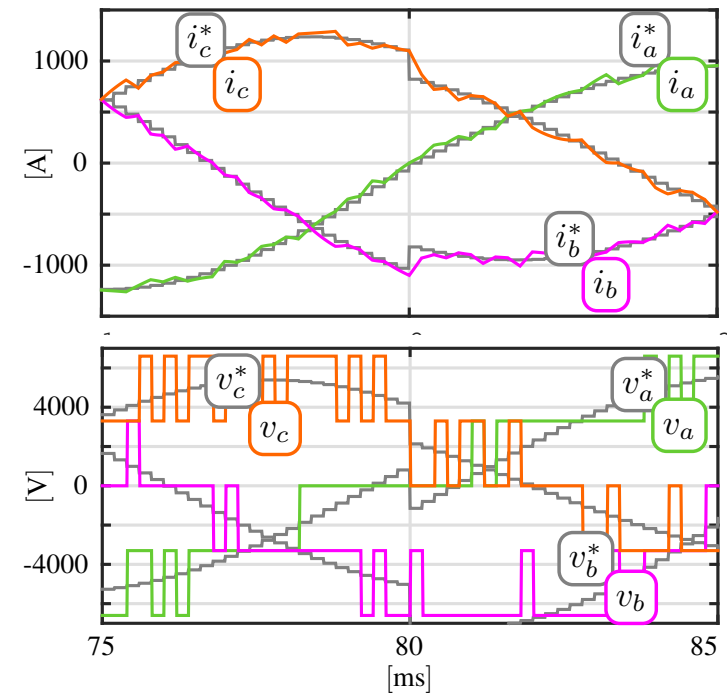

Fig. 3. Zoom of Fig. 2 for $i_{a b c}, v_{a b c}$ between $75-85 \mathrm{~ms}$

error. Once this is achieved, i.e. $x(k) \approx x^{\star}(k)$, the first part of the cost function is reduced, becoming comparable to the second term. This yields to an optimal control input, $u(k)$, that minimizes the current error, while tracking the input reference, $u^{\star}(k)$, during the steady-state. This input reference accounts for the required common-mode voltage a per (25). As a result of this proper tuning, the predictive controller is extracting now unbalanced power from the $\mathrm{CHB}$ converter while injecting balanced power to the grid. The steady-state values of the CHB currents, voltages, and powers per phase are included in Table II.

\section{CONCLUSION}

In this work, a predictive controller for CHB converter has been proposed. This predictive control strategy is able to extract unbalanced power from the dc-source while injecting balanced power to the grid, as verified by the simulation results. This has been achieved by including an input tracking error in the proposed cost function. The proper selection of the weighting factor allows one to preserve the well-known fast dynamic response of the predictive controller during transients 
and achieve power balance in the steady state.

\section{REFERENCES}

[1] S. Kouro, M. Malinowski, K. Gopakumar, J. Pou, L. Franquelo, B. Wu, J. Rodríguez, M. A. Pérez, and J. Leon, "Recent Advances and Industrial Applications of Multilevel Converters," IEEE Transactions on Industrial Electronics, vol. 57, no. 8, pp. 2553-2580, 2010.

[2] E. Al-nabi, B. Wu, N. Zargari, and V. Sood, "Input Power Factor Compensation for High-Power CSC Fed PMSM Drive Using d -Axis Stator Current Control," IEEE Transactions on Dielectrics and Electrical Insulation, vol. 59, no. 2, pp. 752-761, 2012.

[3] P. Acuna, L. Moran, M. Rivera, R. P. Aguilera, R. Burgos, and V. G. Agelidis, "A Single-Objective Predictive Control Method for a Multivariable Single-Phase Three-Level NPC Converter-Based Active Power Filter," IEEE Transactions on Industrial Electronics, vol. 62, no. 7, pp. 4598-4607, 2015.

[4] J. Carrasco, L. Franquelo, J. Bialasiewicz, E. Galvan, R. Guisado, M. Prats, J. Leon, and N. Moreno-Alfonso, "Power-Electronic Systems for the Grid Integration of Renewable Energy Sources: A Survey," IEEE Transactions on Industrial Electronics, vol. 53, no. 4, pp. 1002-1016, 2006.

[5] R. P. Aguilera, P. Lezana, and D. E. Quevedo, "Switched Model Predictive Control for Improved Transient and Steady-State Performance," IEEE Transactions on Industrial Informatics, vol. 11, no. 4, pp. 968977, 2015.

[6] C. D. Townsend, T. Summers, J. Vodden, A. J. Watson, R. E. Betz, and J. Clare, "Optimization of Switching Losses and Capacitor Voltage Ripple Using Model Predictive Control of a Cascaded H-Bridge Multilevel StatCom," IEEE Transactions on Power Electronics, vol. 28, no. 7, pp. 3077-3087, 2013.

[7] M. A. Pérez, S. Bernet, J. Rodríguez, S. Kouro, and R. Lizana, "Circuit Topologies, Modeling, Control Schemes, and Applications of Modular Multilevel Converters," IEEE Transactions on Power Electronics, vol. 30, no. 1, pp. 4-17, 2015.

[8] S. Rivera, S. Kouro, B. Wu, J. Leon, J. Rodríguez, and L. Franquelo, "Cascaded H-bridge multilevel converter multistring topology for large scale photovoltaic systems," in 2011 IEEE 20th International Symposium on Industrial Electronics (ISIE). IEEE, 2011, pp. 1837-1844.

[9] Y. Yu, G. Konstantinou, B. Hredzak, and V. Agelidis, "Power Balance of Cascaded H-Bridge Multilevel Converters for Large-scale Photovoltaic Grid Integration," IEEE Transactions on Power Electronics, no. 99, p. 1 , 2015.

[10] Y. Yu, G. Konstantinou, B. Hredzak, and V. G. Agelidis, "Power Balance Optimization of Cascaded H-Bridge Multilevel Converters for Large-scale Photovoltaic Integration," IEEE Transactions on Power Electronics, vol. PP, no. 9, pp. 1-1, Jun. 2009.
[11] J. Rodríguez, M. P. Kazmierkowski, J. Espinoza, P. Zanchetta, H. AbuRub, H. A. Young, and C. A. Rojas, "State of the Art of Finite Control Set Model Predictive Control in Power Electronics," IEEE Transactions on Industrial Informatics, vol. 9, no. 2, pp. 1003-1016, May 2013.

[12] S. Vazquez, J. Leon, L. Franquelo, J. Rodríguez, H. A. Young, A. Marquez, and P. Zanchetta, "Model Predictive Control: A Review of Its Applications in Power Electronics," IEEE Industrial Electronics Magazine, vol. 8, no. 1, pp. 16-31, 2014.

[13] D. E. Quevedo, R. P. Aguilera, and T. Geyer, "Predictive Control in Power Electronics and Drives: Basic Concepts, Theory, and Methods," in Studies in Computational Intelligence. Cham: Springer International Publishing, Jan. 2014, pp. 181-226.

[14] S. Vazquez, A. Marquez, R. Aguilera, D. Quevedo, J. Leon, and L. Franquelo, "Predictive Optimal Switching Sequence Direct Power Control for Grid-Connected Power Converters," IEEE Transactions on Industrial Electronics, vol. 62, no. 4, pp. 2010-2020, 2015.

[15] P. Lezana, R. P. Aguilera, and D. E. Quevedo, "Model Predictive Control of an Asymmetric Flying Capacitor Converter," IEEE Transactions on Industrial Electronics, vol. 56, no. 6, pp. 1839-1846, 2009.

[16] D. E. Quevedo, R. P. Aguilera, M. A. Pérez, P. Cortés, and R. Lizana, "Model Predictive Control of an AFE Rectifier With Dynamic References," IEEE Transactions on Power Electronics, vol. 27, no. 7, pp. 3128-3136, 2012.

[17] R. P. Aguilera, P. Lezana, and D. E. Quevedo, "Finite-Control-Set Model Predictive Control With Improved Steady-State Performance," IEEE Transactions on Industrial Informatics, vol. 9, no. 2, pp. 658-667, May 2013.

[18] J. D. Barros, J. F. A. Silva, and E. G. A. Jesus, "Fast-Predictive Optimal Control of NPC Multilevel Converters," IEEE Transactions on Industrial Electronics, vol. 60, no. 2, pp. 619-627, 2013.

[19] V. Yaramasu, M. Rivera, M. Narimani, B. Wu, and J. Rodríguez, "Model Predictive Approach for a Simple and Effective Load Voltage Control of Four-Leg Inverter With an Output $L C$ Filter," IEEE Transactions on Industrial Electronics, vol. 61, no. 10, pp. 5259-5270, 2014.

[20] P. R. Martinez-Rodriguez, G. Escobar, A. A. Valdez-Fernandez, M. Hernandez-Gomez, and J. M. Sosa, "Direct Power Control of a Three-Phase Rectifier Based on Positive Sequence Detection," IEEE Transactions on Industrial Electronics, vol. 61, no. 8, pp. 4084-4092, 2014.

[21] P. Cortés, A. Wilson, S. Kouro, J. Rodríguez, and Abu-Rub, "Model Predictive Control of Multilevel Cascaded H-Bridge Inverters," IEEE Transactions on Industrial Electronics, vol. 57, no. 8, pp. 2691-2699, 2010.

[22] R. P. Aguilera and D. E. Quevedo, "Predictive Control of Power Converters: Designs With Guaranteed Performance," IEEE Transactions on Industrial Informatics, vol. 11, no. 1, pp. 53-63, 2015. 